\title{
ASYMPTOTIC DEVELOPMENTS FOR A BOUNDARY VALUE PROBLEM CONTAINING A PARAMETER*
}

\author{
BY \\ RICHARD C. MACCAMY \\ Carnegie Institute of Technology
}

1. Introduction. It is the purpose of this paper to point out a curious type of singularity which can arise in the perturbation of the solutions of boundary value problems containing a parameter. We shall consider two such problems each for functions of $x$ and $y$ continuous in $y \leq 0,(x, y)$ bounded, and harmonic in $y<0$. The two functions satisfy the following sets of boundary condition, $K$ being a positive constant and $g(x)$ a given function:

$\begin{array}{llll}\text { Problem I } & \left(A_{\mathrm{I}}\right) u_{\nu}(x, 0)=0 & \text { for } & |x|>1 \\ & \left(B_{\mathrm{I}}\right) u_{\nu}(x, 0)+K u(x, 0)=g(x) & \text { for } & |x|<1, \\ \text { Problem II } & \left(A_{\mathrm{II}}\right) u_{\nu}(x, 0)-K u(x, 0)=0 & \text { for } & |x|>1 \\ & \left(B_{\mathrm{II}}\right) u_{\nu}(x, 0)=g(x) & \text { for } & |x|<1 .\end{array}$

We admit the possibility of the function $g(x)$ depending on $K$ provided it be analytic in $K$ for $K$ sufficiently small. The two problems clearly have much the same character, the one deriving from the other essentially by interchanging the roles of the intervals $|x|<1$ and $|x|>1$. In fact it can be shown that they are equivalent, that is solution of the one yields the solution of the other [1]. The two problems indicate strikingly the need for caution in the study of perturbations for despite their apparent similarity we shall find entirely different behavior of the solutions for small $K$.

Physically (I) is a problem in heat conduction while (II) governs the diffraction of surface water waves by a rigid dock of finite width [2]. Differences in the problem become more apparent when we specify the behavior of the solutions of (I) and (II) for large $x^{2}+y^{2}$. It is easily seen in fact that the following behaviors hold for (I) and (II) respectively, [2].

$$
\begin{aligned}
& \left(C_{\mathrm{I}}\right) \quad u(x, y)-c \log \left(x^{2}+y^{2}\right)=0\left(x^{2}+y^{2}\right)^{-1} \quad \text { as } x^{2}+y^{2} \rightarrow \infty, \quad c \text { constant } \\
& \left(C_{\mathrm{II}}\right) \quad u(x, y)-T e^{K y} e^{i K x}=0\left(\frac{1}{x}\right) \text { as } x \rightarrow+\infty, \quad y \text { bounded } \\
& u(x, y)-R e^{K y} e^{i K x}=0\left(\frac{1}{x}\right) \text { as } x \rightarrow-\infty, \quad y \text { bounded, }
\end{aligned}
$$

for some constants $T$ and $R$.

If one sets $K=0$ in (I) and (II) the problems become formally the same namely

$$
\left(A_{0}\right) \quad u_{y}(x, 0)=0 \quad \text { for }|x|>1
$$

${ }^{*}$ Received May 9, 1958. This work was supported by the Office of Naval Research, Contract Nonr222(25). 


$$
\left(B_{0}\right) \quad u_{y}(x, 0)=g(x) \text { for }|x|<1 .
$$

This last problem is trivial having as solution the function*,

$$
u_{0}(x, y)=-\frac{1}{\pi} \int_{-1}^{+1} g(t) \log \left[(x-t)^{2}+y^{2}\right] d t .
$$

One may ask now whether the solutions of problems (I) and (II) tend to $u_{0}(x, y)$ as $K \rightarrow 0$. A glance at conditions $\left(C_{\mathrm{I}}\right)$ and $C_{\mathrm{II}}$ ) leads one to suspect that such is the case for the solution of (I) while for the solution of (II) difficulties might arise, a suspicion which proves correct. We shall see that the solution of (I) is in fact an analytic function, $u(x, y ; K)$ for complex $K$ of sufficiently small absolute value, with $u(x, y ; 0)=u_{0}(x, y)$. The solution of (II) is also analytic in $K$ but for a region $0<|K|<\rho$. It has in fact the rather curious asymptotic expansion, $\dagger$

$$
K u(x, y ; K) \sim \sum_{m=0}^{\infty} \sum_{n=0}^{\infty} A_{m n}(x, y) K^{m}(K \log K)^{n} \quad A_{00} \equiv 0 .
$$

Thus the solution of (II) does not possess a limit as $K \rightarrow 0$. The apparent physical paradox introduced in the finite dock problem can be removed by more careful attention to physical units but the odd development (1.2) for the solution of (II) remains.

2. Developments for Problem (I). The development of the solution of Problem (I) for small $K$ is elementary. To solve (I) we try the function,

$$
u(x, y ; K)=-\frac{1}{\pi} \int_{-1}^{+1} f(t ; K) \log \left[(x-t)^{2}+y^{2}\right] d t .
$$

This function clearly is harmonic in $y<0$ and satisfies $\left(A_{\mathrm{I}}\right)$ and $\left(C_{\mathrm{I}}\right)$. By a well known formula, it will satisfy $\left(B_{\mathrm{I}}\right)$ provided $f(t ; K)$ is a solution of the integral equation,

$$
f(x ; K)+\frac{K}{\pi} \int_{-1}^{+1} f(t ; K) \log |x-t| d t=g(x) \quad|x|<1 .
$$

The usual method of successive approximations establishes that (2.2) possesses a solution $f(x ; K)$ for suitably small $K$ which is analytic in $K$. Further we have $f(x, 0)=g(x)$. Substitution in (2.1) yields a solution $u(x, y ; K)$ of (I) analytic for $K$ sufficiently small and with $u(x, y ; 0)=u_{0}(x, y)$.

3. Developments for Problem (II). An integral representation for Problem (II) can be obtained in the usual manner by introducing the Green's function $G(x, y, t ; K)$ defined by,

$$
\begin{aligned}
G(x, y, t ; K)=-1 / 2 \log \left[(x-t)^{2}+y^{2}\right] & +\frac{K}{2} e^{K_{\nu}} \int_{\nu}^{\infty} e^{-K \eta} \log \left[(x-t)^{2}+\eta^{2}\right] d \eta \\
& +\pi i e^{K_{\nu}} e^{i K|x-t|} .
\end{aligned}
$$

We have,

$$
G_{y}=K G=-\frac{y}{(x-t)^{2}+y^{2}}
$$

Thus setting,

${ }^{*}$ For this we need Hölder continuity of $g(x)$.

$\dagger$ It is to be noted that asymptotic developments of the form (1.2) have been observed before in Problem (II) but in an entirely different connection [3]. The meaning of the asymptotic development is made clear in Sec. 3 . 


$$
u(x, y ; K)=-\frac{1}{\pi} \int_{-1}^{+1} f(t ; K) G(x, y, t ; K) d t
$$

we find, in the same manner as (2.2) was obtained from (2.1),

$$
u_{\nu}(x, 0 ; K)=f(x ; K)+\frac{K}{\pi} \int_{-1}^{+1} f(t ; K) G(x, 0, t ; K) d t \quad \text { on } \quad|x|<1
$$

while $u(x, y ; K)$ satisfies $\left(A_{\mathrm{II}}\right)$ for any choice of $f$. It is further easy to show that the function defined by (3.2) is harmonic in $y<0$, continuous in $y \leq 0$ and satisfies $\left(C_{\mathrm{II}}\right)$. Thus (3.2) yields a solution of Problem (II) if $f(x ; K)$ is a solution of the integral equation,

$$
g(x)=f(x ; K)+\frac{K}{\pi} \int_{-1}^{+1} f(t ; K) G(x, 0, t ; K) d t \quad \text { on }|x|<1 .
$$

Note that the function $f(x)$ is related to the solution $u$ by,

$$
f(x ; K)=u_{y}(x, 0 ; K)-K u(x, 0 ; K) .
$$

In order to express $u(x, y ; K)$ as a function of the parameter $K$ we must so express $G(x, y, t ; K)$. Observe first that,

$$
\begin{gathered}
\pi i e^{K_{\nu}} e^{i K|x-t|}=\sum_{m=0}^{\infty} A_{m}(x-t, y) K^{m}, \\
K e^{K \nu} \int_{\nu}^{0} e^{-K \eta} \log \left[(x-t)^{2}+\eta^{2}\right] d \eta \\
=K e^{K \nu} \sum_{m=1}^{\infty} \frac{(-1)^{n} K^{n}}{n !} \int_{\nu}^{0} \eta^{n} \log \left[(x-t)^{2}+\eta^{2}\right] d \eta=\sum_{m=1}^{\infty} B_{m}(x, y, t) K^{m},
\end{gathered}
$$

the series converging absolutely and uniformly for bounded $x, y, t$, and $|K|$ The term,

$$
I=K e^{K \nu} \int_{0}^{\infty} e^{-K \eta} \log \left[(x-t)^{2}+\eta^{2}\right] d \eta
$$

is more complicated. Note first that,

$$
\begin{aligned}
I=e^{K_{\nu}} \int_{0}^{\infty} e^{-\tau} \log \left[(x-t)^{2}\right. & \left.+\frac{\tau^{2}}{K^{2}}\right] d \tau \\
& =-2 e^{K_{\nu}} \log K+e^{K_{\nu}} \int_{0}^{\infty} e^{-\tau} \log \left[(K|x-t|)^{2}+\tau^{2}\right] d \tau,
\end{aligned}
$$

hence,

$$
\lim _{K \rightarrow 0}(I+2 \log K)=2 \int_{0}^{\infty} e^{-t} \log t d t=-2 \gamma,
$$

where $\gamma$ is Euler's constant.

To obtain a more detailed description of $I$ we study it as a function of complex $K$. The integral (3.6) defining $I$ converges, and yields an analytic function as long as $0<|K|, 0 \leq \arg K<\pi / 2 . I$ may be continued analytically to a larger sector by shifting the path of integration. In order to continue $I(K)$ into $\arg K \geq \pi / 2$ we shift the path into the sector $\arg \eta<0$ so that it keeps the points $\pm i|x-t|$ always to the right and runs to $\infty$ along the ray $\arg \eta=-\arg K$, thus keeping $K \eta$ positive for large $|\eta|$. 
In particular if $I^{+}$denotes the value of $I$ after one counter-clockwise circuit of the origin we have,

$$
I^{+}=K e^{K \nu} \int_{c} e^{-K \eta} \log \left[(x-t)^{2}+\eta^{2}\right] d \eta+K e^{K \nu} \int_{0}^{\infty} e^{-K \eta}\left(\log \left[(x-t)^{2}+\eta^{2}\right)\right]^{-} d \eta,
$$

where $c$ denotes a closed path surrounding $\pm i|x-t|$ in the negative sense and (log $\left.\left[(x-t)^{2}+\eta^{2}\right]\right)^{-}$denotes the value of the logarithm after $\eta$ circles the points $\pm i|x-t|$ in the negative sense. Hence,

$$
\begin{array}{r}
I^{+}-I=2 K e^{K \nu}\left\{\pi i \int_{0}^{-i|x-t|} e^{-K \eta} d \eta+\pi i \int_{0}^{i|x-t|} e^{-K \eta} d \eta-2 \pi i \int_{0}^{\infty} e^{-K \eta} d \eta\right\} \\
=-4 \pi i e^{K_{\nu}} \cos K|x-t| .
\end{array}
$$

Equations (3.7) and (3.8) together imply that $I(x, y, t)$ has the form,

$$
I=\left\{-2 e^{K_{\nu}} \cos K|x-t|\right\} \log K+\sum_{m=0}^{\infty} c_{m}(x, y, t) K^{m},
$$

where by (3.7) $c_{0}=-2 \gamma$. Combining (3.5) and (3.9) we have finally,

$$
\begin{aligned}
G(x, y, t ; K) & =\left\{-e^{K y} \cos K|x-t|\right\} \log K+\sum_{m=0}^{\infty} G_{m}(x, y, t) K^{m} \\
& =-\log K \sum_{m=0}^{\infty} H_{m}(x, y, t) K^{m}+\sum_{m=0}^{\infty} G_{m}(x, y, t) K^{m}
\end{aligned}
$$

the series converging for suitably small $K$. For reference we write down the first few terms:

$$
\begin{aligned}
H_{0}=-1 \quad & H_{1}=-y \quad H_{2}=-\frac{1}{2}|x-t|^{2}+\frac{1}{2} y^{2}, \\
G_{0}= & (\pi i-\gamma)-\log \left[(x-t)^{2}+y^{2}\right]^{1 / 2} .
\end{aligned}
$$

For the case $y=0$ all of the terms in the development may be obtained from formulae given in [4].

With the developments (3.10) at hand we are ready to discuss $u(x, y ; K)$. Suppose $g(x)=\sum_{m=0}^{\infty} g_{m}(x) K^{m}$. Then substituting (3.10) in (3.3) we have,

$$
\begin{aligned}
f(x ; K) & -\frac{1}{\pi} \sum_{m=0}^{\infty} K^{m}(K \log K) \int_{-1}^{+1} f(t) H_{m}(x, 0, t) d t \\
& +\frac{1}{\pi} \sum_{m=0}^{\infty} K^{m+1} \int_{-1}^{+1} f(t) G_{m}(x, 0, t) d t=\sum_{m=0}^{\infty} g_{m}(x) K^{m} \quad \text { on } \quad|x|<1 .
\end{aligned}
$$

Now we remark that the power products $K^{m}(K \log )^{n}$ can be ordered according to increasing degree of vanishing as $K \rightarrow 0$, i.e.

$\left.\left.\lim _{K \rightarrow 0} \frac{K^{m^{\prime}}(K}{K^{m}(K} \log K\right)^{n^{\prime}} \log K\right)^{n^{-}}=0$ if $m^{\prime}+n^{\prime}>m+n$ or $m^{\prime}+n^{\prime}=m+n$ and $n>n^{\prime}$.

We proceed to develop the solution of (3.11) in the set of these power products. Passing to limit $K=0$ in (3.11) we find,

$$
f_{00}(x)=\lim _{K \rightarrow 0} f(x ; K)=g_{0}(x) .
$$

Then (3.11) becomes, 


$$
f(x ; K)-f_{00}(x)-\frac{1}{\pi} K \log K \int_{-1}^{+1} f(t ; k) H_{0}(x, 0, t) d t=o(K \log K) \quad \text { on } \quad|x|<1 .
$$

Dividing by $K \log K$ and passing to limit $K=0$,

$$
f_{01}(x)=\lim _{K \rightarrow 0}\left[\frac{f(x ; K)-f_{00}(x)}{K \log K}\right]=\frac{1}{\pi} \int_{-1}^{+1} f_{00}(t) d t=\frac{1}{\pi} \int_{-1}^{+1} g_{0}(t) d t .
$$

Reentering in (3.11),

$f(x ; K)-f_{00}(x)-f_{01}(x) K \log K+\frac{K}{\pi} \int_{-1}^{+1} f(t ; k) G_{0}(x, 0, t)$

$$
=g_{1}(x) K+o(K) \quad \text { on }|x|<1 .
$$

Dividing by $K$ and passing to limit $K=0$,

$f_{10}(x)=\lim _{K \rightarrow 0}\left[f(x ; K)-f_{00}(x)-f_{01}(x) K \log K\right] / K=g_{1}(x)-\frac{1}{\pi} \int_{-1}^{+1} f_{00}(t) G_{0}(x, 0, t) d t$.

We have indicated here the first three steps of a process which can be continued indefinitely. The process leads ultimately to,

$$
f(x ; K) \sim \sum_{m=0}^{\infty} \sum_{n=0}^{\infty} f_{m n}(x) K^{m}(K \log K)^{n} .
$$

That the successive $f_{m n}(x)$ can be determined by recursion one sees as follows. We say $f(x ; K)$ has an estimate of degree $(m, n)$ if,

$$
f(x ; K)=P(K, K \log K)+o\left(K^{m}(K \log K)^{n}\right),
$$

where $P$ is a polynomial (with coefficients depending on $x$ ) of degree $(m, n)$. Since,

$$
K G=-K \log K+o(K \log K)
$$

we see that the product of $K G$ with a polynomial of degree $(m, n)$ is a polynomial of degree $(m, n+1)$ plus terms $o\left(K^{m}(K \log K)^{n+1}\right)$. Suppose then that we have shown $f(x ; K)$ to have an estimate of degree $(m, n)$, that is have computed $f_{00}, f_{01}, \cdots, f_{m n}$ in (3.12). Substituting this estimate in the integral in (3.11) we obtain a polynomial of degree $(m, n+1)$ plus terms of o $\left(\left[K^{m}(K \log K)^{n+1}\right]\right.$, with coefficients determined by the known quantities, $f_{00}, \cdots, f_{m n}$. Right hand side of (3.11) has estimates of degree $(m, 0)$ for all $m$, hence (3.11) yields for $f(x ; K)$ an estimate of degree $(m, n+1)$.

Substituting the series (3.12) and (3.10) in the integral representation (3.2) it is readily seen that $u(x, y ; K)$ has the form,

$$
K u(x, y ; K) \sim \sum_{m=0}^{\infty} \sum_{n=0}^{\infty} u_{m}(x, y) K^{m}(K \log K)^{n} .
$$

4. Convergence of the asymptotic developments.* The development (3.13) is asymptotic and the question arises as to whether the series actually converges to yield the function $u(x, y ; K)$. For the special case $g(x)=-e^{i K x}$, which governs the diffraction of waves by a rigid dock, an alternative procedure exists for obtaining the development (3.13). This second technique answers the convergence question while at the same time pointing up the relations which exist between the coefficients of the development.

*The idea of this section was suggested to the author by Professor Hans Lewy. 
For simplicity we consider only the even part of the solution that is the solution of Problem (II) with $g(x)=-\cos K x$. The odd part could be treated in the same manner. The solution has the representation (3.2) with $f(x ; K)$ a solution of the equation,

$$
f(x ; K)+\frac{K}{\pi} \int_{-1}^{+1} f(t ; K) G(x, 0, t ; K) d t=-\cos K x \quad \text { on } \quad x<1 .
$$

$G(x, 0, t ; K)$ is defined as a function of complex $K$ by (3.10), that is,

$$
G(x, 0, t ; K)=-\{\cos K|x-t|\} \log K+\sum_{m=0}^{\infty} G_{m}(x, 0 ; t) K^{m} .
$$

The usual method of successive approximations applied to (4.1) shows that the solution, as a uniform limit of functions analytic in $K$, is analytic for $|K| \neq 0$ sufficiently small. Let $K^{+}, K^{-}$denote the image points of $K$ on a logarithmic Riemann surface of $K$ after positive and negative circuits respectively of the origin. By (4.2),

$$
G\left(x, 0, t ; K^{+}\right)-G(x, 0, t ; K)=-2 \pi i \cos K|x-t|
$$

hence if we form (4.1) for $K^{+}$we find,

$$
\begin{aligned}
f\left(x, K^{+}\right)+\frac{K}{\pi} \int_{-1}^{+1} f\left(t, K^{+}\right) & G(x, 0, t ; K) d t \\
& -2 i K \int_{-1}^{+1} f\left(t, K^{+}\right) \cos K|x-t| d t=-\cos K x .
\end{aligned}
$$

Subtracting (4.1) from (4.3) and noting that $f$ is even with respect to $t$

$$
\begin{aligned}
f\left(x, K^{+}\right)- & f(x, K)+\frac{K}{\pi} \int_{-1}^{+1}\left[f\left(t, K^{+}\right)-f(t, K)\right] G(x, 0, t ; K) d t \\
& =2 i K \int_{-1}^{+1} f\left(t, K^{+}\right) \cos K|x-t| d t=2 i K \cos K x \int_{-1}^{+1} f\left(t, K^{+}\right) \cos K t d t
\end{aligned}
$$

hence the difference, $f\left(x, K^{+}\right)-f(x, K)$ satisfies, except for a multiplicative constant the same integral equation (4.1), as $f(x, K)$ itself. Therefore,

$$
f\left(x, K^{+}\right)-f(x, K)=2 i K f(x, K) \int_{-1}^{+1} f\left(t, K^{+}\right) \cos K t d t
$$

or,

$$
f(x, K)-f\left(x, K^{-}\right)=2 i K f\left(x ; K^{-}\right) \int_{-1}^{+1} f(t, K) \cos K t d t .
$$

The function $f(x, K)$ thus satisfies a non-linear difference equation in $K$. If we let,

$$
\lambda(K)=\int_{-1}^{+1} f(t, K) \cos K t d t
$$

we find by multiplying (4.4) by $\cos K x$ and integrating,

$$
\lambda(K)-\lambda\left(K^{-}\right)=2 i K \lambda(K) \lambda\left(K^{-}\right) .
$$

This difference equation for $\lambda(K)$ is still non-linear but we can form from it a linear equation. As in the derivation of (3.12) we have from (4.1), 


$$
f(t, K)=-1+o(1) \text { as } K \rightarrow 0 \text {. }
$$

Hence,

$$
\lambda(K)=-2+o(1) \text { as } K \rightarrow 0
$$

so that $\lambda(K)$ [and also $\lambda\left(K^{-}\right)$] is different from 0 for $K$ sufficiently small. Thus for small $K,(4.5)$ can be divided by $\lambda(K) \lambda\left(K^{-}\right)$to yield,

$$
\frac{1}{\lambda\left(K^{-}\right)}-\frac{1}{\lambda(K)}=2 i K \text {. }
$$

It follows that,

$$
\tau \equiv \frac{1}{\lambda(K)}=-\frac{K}{\pi} \log K+S(K) \quad \text { or } \quad \lambda(K)=\frac{1}{S(K)-\frac{K}{\pi} \log K},
$$

where $S(K)$ is single-valued, and being bounded by (4.6), is a power series, i.e.,

$$
S(K)=\frac{1}{2}\left(1+\sum_{n=1}^{\infty} S_{n} K^{n}\right)
$$

Substituting (4.7) in (4.4) we find,

$$
f(x, K)-\left[1+\frac{2 i K}{S(K)-\frac{K}{\pi} \log K}\right] f\left(x, K^{-}\right)=0 .
$$

Now observe that,

$$
\tau\left(K^{-}\right)=S(K)-\frac{K}{\pi} \log K+2 i K=\tau(K)\left[1+\frac{2 i K}{S(K)-\frac{K}{\pi} \log K}\right] .
$$

Also $\tau(0) \neq 0$, hence for sufficiently small $K(4.9)$ can be written

$$
\tau(K) f(x, K)-\tau\left(K^{-}\right) f\left(x, K^{-}\right)=0
$$

i.e., $\tau(K) f(x, K)$ is a single-valued function, $p(x ; K)$, which is continuous at $K=0$, and thus,

$$
f(x, K)=\frac{p(x, K)}{S(K)-\frac{K}{\pi} \log K} \quad p(x, K)=\sum_{n=0}^{\infty} p_{n}(x) K^{n} .
$$

From (4.10) two interesting facts may be observed. First we can write, since $S(0)=\frac{1}{2}$,

$$
f(x, K)=2 p(x ; K) \frac{1}{1-Z(K)},
$$

where

$$
Z(K)=2 \frac{K}{\pi} \log K-\sum_{n=1}^{\infty} S_{n} K^{n} .
$$

Since $|Z(K)|<1$ for $K$ sufficiently small, say $|K|<K_{0}$, we have, 


$$
f(x, K)=2 p(x ; K) \sum_{n=0}^{\infty}(Z(K))^{n}
$$

which it is readily seen leads to a convergent double power series in $K$ and ( $K \log K$ ).

Second we see by (4.10) and (4.11) that $f(x, K)$ is completely determined by the two analytic functions $p(x, K)$ and $S(K)$. That is to say in the double series development

$$
f(x, K)=\sum_{m=0}^{\infty} \sum_{n=0}^{\infty} a_{m n}(x) K^{m}(K \log K)^{n}
$$

for $f(x, K)$ there are at most two simple infinities of the coefficients $a_{m n}$ which are sufficient to determine the others. In reality the analytic functions $p(x ; K)$ and $S(K)$ are related so that only a single infinity of the coefficients need be determined. One sees this by using (4.11) to express the $S_{n}$ and $p_{n}(x)$ as functionals of $f(x: K)$, i.e., of the $a_{i j}(x)$. If the operation in (4.11) is carried out and the series (4.12) is substituted on the left, we obtain a series of relations, the first few of which we write down here:

$$
\begin{gathered}
2 p_{0}(x)=a_{00}(x), \\
\frac{4 p_{0}(x)}{\pi}=a_{01}(x), \\
2 p_{1}(x)-2 p_{0}(x) S_{1}=a_{10}(x), \\
\frac{8}{\pi^{2}} p_{0}(x)=a_{02}, \\
\frac{4 p_{1}(x)}{\pi}-\frac{8 S_{1} p_{0}(x)}{\pi}=a_{11}(x) .
\end{gathered}
$$

We see from these relations first that the $p_{n}(x)$ are determined by the $a_{i 0}(x)$ and the $S_{n}$ and then that the $a_{i j}(x)$ for $j \geq 1$ are determined by the $a_{i 0}(x)$. The $p_{n}(x)$ and $\left\{S_{n}\right\}$ and hence $f(x, K)$ are completely specified if one knows the values of the $a_{i 0}(x) i=0$, $1,2, \cdots$.

The intermediate step of computing the $a_{i}(x)$ may be eliminated as follows. Substituting (4.10) and (3.10) in (4.1) yields,

$$
\begin{aligned}
p(x, K)+\frac{1}{\pi} \int_{-1}^{+1} p(t, K)\{-K \log K( & \left.\cos K|x-t|)+\sum_{m=0}^{\infty} G_{m}(x, 0, t) K^{m+1}\right\} d t \\
& =-\cos K x\left[S(K)-\frac{K}{\pi} \log K\right] \text { on }|x|<1 .
\end{aligned}
$$

We can equate here terms which involve $\log K$ and those which do not, obtaining

$$
\begin{gathered}
-\frac{1}{\pi} \int_{-1}^{+1} p(t, K) \cos K|x-t| d t=\frac{1}{\pi} \cos K x \text { on }|x|<1 \\
p(x, K)+\frac{1}{\pi} \int_{-1}^{+1} p(t, K) \sum_{m=0}^{\infty} G_{m}(x, 0, t) K^{m+1} d t=-S(K) \cos K x .
\end{gathered}
$$

Since $p(x, K)$ is an even function of $x$ (4.13) may be rewritten,

$$
\int_{-1}^{+1} p(t, K) \cos K t d t=-1
$$


Set $p^{*}(x, K)=p(x, K) / S(K)$. Recalling that $S(0) \neq 0 p^{*}(x, K)$ is an analytic function of $K$ for $K$ small, i.e.,

$$
p^{*}(x, K)=\sum_{n=0}^{\infty} p_{n}^{*}(x) K^{n}
$$

Substituting in (4.14) and equating coefficients of $K^{r}, r=0,1,2, \cdots$, yields, $p_{0}^{*}=-1$,

$$
\begin{aligned}
& p_{r}^{*}(x)+\frac{1}{\pi} \sum_{n=0}^{r-1} \int_{-1}^{+1} p_{n}(t) G_{r-1-n}(x, 0, t) d t \\
& =\frac{(-1)^{r+1}}{2 r !} x^{2 r} \text { if } r \text { is even, } \quad r>0 \\
& =0 \quad \text { if } r \text { is odd. }
\end{aligned}
$$

Equation (4.16) determines the $p_{r}(x)$ recursively. For example:

$$
\begin{gathered}
p_{0}^{*}(x)=-1, \\
p_{1}^{*}(x)=-\frac{1}{\pi} \int_{-1}^{+1} p_{0}^{*}(t) G_{0}(x, 0, t) d t=\frac{2}{\pi}(\pi i-\gamma), \\
-\frac{1}{\pi}[(1+x) \log (1+x)+(1-x) \log (1-x)-2 x] .
\end{gathered}
$$

Once the $p_{r}^{*}(x)$ are found Eq. (4.15) can be used to determined $S(K)$. Substituting $p(x, K)=S(K) p^{*}(x ; K)$ in (4.15) and equating coefficients of $K^{i}$ yields,

$$
\begin{aligned}
\frac{1}{2} \sum_{m=0}^{[i / 2]} \frac{(-1)^{m}}{2 m !} \int_{-1}^{+1} p_{i-2 m}^{*}(t) t^{2 m} d t^{*} \\
\quad+\sum_{n=1}^{i} \sum_{m=0}^{[i-n / 2]} S_{n} \frac{(-1)^{m}}{2 m !} \int_{-1}^{+1} p_{i-n-2 m}^{*} t^{2 m} d t=0, \quad i=1,2,3, \cdots .
\end{aligned}
$$

Equation (4.17) determines the $S_{n}$ recursively since the coefficient of $S_{i}$ is,

$$
\int_{-1}^{+1} p_{0}^{*}(t) d t=-2 \neq 0
$$

For example:

$$
S_{1}=\frac{1}{4} \int_{-1}^{+1} p_{1}^{*}(t) d t=i-\frac{\gamma}{\pi}-\frac{1}{2}(\log 2-1) .
$$

Once $S(K)$ and $p^{*}(x ; K)$ are found, $p(x ; K)$ is obtained as their product.

\section{REFERENCES}

1. R. C. MacCamy, The Principle of Babinet, Can. J. Math. (to appear)

2. Hanan Rubin, The dock of finite extent, Communs. Pure and Appl. Math. VII, No. 2, 317-344 (1954)

3. Hans Lewy, Developments at the confluence of analytic boundary conditions, University of California Publications in Mathematics, vol. I, No. 7, 247-280 (1950)

4. R. C. MacCamy, Une solution par potentiel de sources pour l'équation des houles a courtes crêtes, La Houille Blanche, Numero 3 (1957)

\footnotetext{
* [z] denotes greatest integer not greater than $z_{0}$
} 